\title{
Plasma B-Type Natriuretic Peptide Level and Cardiovascular Events in Chronic Kidney Disease in a Community-Based Population
}

\author{
Masafumi Sakuma, MD; Motoyuki Nakamura, MD; Fumitaka Tanaka, MD; \\ Toshiyuki Onoda, MD*; Kazuyoshi Itai, PhD*; Kozo Tanno, MD*; Masaki Ohsawa, MD*; \\ Kiyomi Sakata, MD*; Yuki Yoshida, MD**; Kazuko Kawamura, MS ${ }^{\dagger}$; \\ Shinji Makita, MD; Akira Okayama, MD ${ }^{\dagger \dagger}$
}

\begin{abstract}
Background: Plasma B-type natriuretic peptide (BNP) levels are confounded by renal dysfunction, so this study examined whether plasma BNP might be a reliable biomarker of the onset of cardiovascular (CV) events in a population-based cohort with impaired renal function.
\end{abstract}

\begin{abstract}
Methods and Results: Baseline data, including plasma BNP, serum creatinine, and urinary protein levels, were determined in participants from a community-based population. Estimated glomerular filtration rate (eGFR) was calculated, and chronic kidney disease (CKD) was defined as either: eGFR $<60 \mathrm{ml} \cdot \mathrm{min}^{-1} \cdot 1.73 \mathrm{~m}^{-2}$ and/or proteinuria (CKD definition-1) or GFR $<60 \mathrm{ml} \cdot \mathrm{min}^{-1} \cdot 1.73 \mathrm{~m}^{-2}$ (CKD definition-2). The CV endpoint was surveyed prospectively. The cohorts were followed for 5,275 person-years for CKD definition-1, and for 4,350 person-years for CKD definition-2. The CV event-free survival rate in the highest BNP quartile in either CKD definition was the lowest among the quartile groups $(\mathrm{P}<0.001)$. In multivariate Cox regression models adjusted by traditional $\mathrm{CV}$ risk factors and atrial fibrillation, relative risk $(\mathrm{RR})$ for $\mathrm{CV}$ events was significantly higher in the highest BNP quartile compared with the lowest BNP quartile (CKD definition-1, RR 3.51, $\mathrm{P}<0.01$ : CKD definition-2, RR 4.67, both $\mathrm{P}<0.01$ ).
\end{abstract}

Conclusions: Plasma BNP level provides strong predictive information about the future onset of CV events in CKD subjects selected from the general population. (Circ $J$ 2010; 74: 792-797)

Key Words: General population; Heart failure; Renal failure; Stroke

C hronic kidney disease (CKD), defined as reduced glomerular filtration rate (GFR) and/or proteinuria, increases the risk of cardiovascular (CV) disease and endstage renal disease. ${ }^{1}$ In population-based studies, the prevalence of CKD has been shown to be $7 \%$ in persons aged more than 30 years and to be increased $23-36 \%$ in persons aged more than 65 years. ${ }^{2}$ The trend in the prevalence of CKD has been speculated to increase over time in line with the recent increasing prevalence of diabetes, obesity, and hypertension. ${ }^{3}$ Several reports have emphasized that early identification and treatment of CKD are necessary to prevent serious outcomes in this disorder. ${ }^{1,4}$ However, considering the large number of persons with $\mathrm{CKD}$ in the general population, it may not be easy to provide pharmacological and non-pharmacological interventions for all stages of CKD. In view of these limitations, it may be practical to select CKD subjects at relatively high risk for $\mathrm{CV}$ diseases from the general population, and then provide treatment to prevent their onset. However, there are no established markers to stratify $\mathrm{CV}$ risk in CKD subjects with mild renal dysfunction, such as stage $3 \mathrm{CKD}$, in the mass screening setting.

\section{Editorial p 632}

Natriuretic peptide family proteins, including B-type natriuretic peptide (BNP) and its N-terminal fragment (NT-pro $\mathrm{BNP}$ ), are released from the heart in response to increased intracardiac pressure, cardiac pump dysfunction, hypertensive ventricular hypertrophy, and myocardial ischemia. In community-based studies, increased circulating levels of BNP and NT-pro BNP have been reported to relate to a high risk of $\mathrm{CV}$ events and mortality. 5,6 The high prevalence of $\mathrm{CV}$ events in the group with elevated plasma levels of BNP and NT-proBNP is believed related to the high prevalence of subclinical heart disease. However, plasma concentrations of BNP and NT-proBNP increase as GFR declines in patients

Received November 6, 2009; accepted December 14, 2009; released online February 17, 2010 Time for primary review: 25 days

Department of Internal Medicine (Cardiology Division), *Department of Preventive Medicine, **Department of Neurosurgery, Iwate Medical University, ${ }^{\dagger}$ Iwate Health Service Association, Morioka and †The First Anti-tuberculosis Association, Tokyo, Japan

Mailing address: Motoyuki Nakamura, MD, Division of Cardiology, Department of Internal Medicine and Memorial Heart Center, Iwate Medical University, 19-1 Uchimaru, Morioka 020-8505, Japan. E-mail: nkmrmoto@iwate-med.ac.jp

ISSN-1346-9843 doi:10.1253/circj.CJ-09-0834

All rights are reserved to the Japanese Circulation Society. For permissions, please e-mail: cj@j-circ.or.jp 
with and without apparent cardiac disorders. ${ }^{7,8}$ In view of these facts, it is unclear whether plasma BNP levels would be a reliable biomarker for predicting $\mathrm{CV}$ events in the cohort of CKD selected from a community-based population.

CKD is usually defined by 2 biomarkers of renal function: urinary protein and reduced GFR. Several community-based studies have applied only the latter definition. ${ }^{9-11}$ However, it is uncertain whether these biomarkers (GFR and urinary protein) provide complementary or overlapping information for $\mathrm{CV}$ risk. Cirillo et al reported that the use of only 1 of the biomarkers underscores the potential to misclassify patients as having or lacking CKD, thus misinterpreting the CV risk. ${ }^{12}$ Therefore, the present study used 2 definitions of CKD to examine whether plasma BNP might be a reliable biomarker for predicting onset of CV diseases in a CKD cohort selected from a community-based general population.

\section{Methods}

\section{Study Population}

The original cohort of the Iwate-KENCO study was recruited from a community-based population living in Ninohe, Kuji, and Miyako districts of northern Iwate prefecture, Japan. The details of the recruitment and measurements of the cohort were shown in previous reports. ${ }^{13,14}$ The total number of participants who agreed to join the Iwate-KENCO study in the 3 districts was 26,469 (original cohort). Of the original cohort living in Ninohe and Kuji districts $(n=15,927), 15,394$ subjects (97\%) had BNP measurements (BNP cohort: men 5,288; women 10,106).

Subjects were excluded from the present analysis for the following reasons: age under $40(n=575)$; history of $\mathrm{CV}$ events, such as myocardial infarction, stroke or heart failure $(n=507)$; missing data of serum creatinine level $(n=28)$, body mass index $(n=47)$, ECG tracing $(n=717)$, or blood pressure $(\mathrm{n}=4)$; estimated GFR $<30 \mathrm{ml} \cdot \mathrm{min}^{-1} \cdot 1.73 \mathrm{~m}^{-2}(\mathrm{n}=28)$. The final statistical analysis included 13,526 subjects (men 4,542; women 8,984). The study protocol was approved by the university ethics committee and local institutional review committees. All participants gave written informed consent.

\section{Definition of CKD}

The eGFR was calculated using an equation from the Modification Diet in Renal Disease Study (MDRD) for the Japanese population. ${ }^{15}$ A urine sample was obtained during a multiphase health examination and urinary protein was semi-quantitatively determined using a dipstick test (Uropaper alpha II, Eiken): proteinuria was defined as trace or more. CKD was defined in the present study in 2 ways: (1) eGFR $<60 \mathrm{ml} \cdot \mathrm{min}^{-1}$. $1.73 \mathrm{~m}^{-2}$ and/or proteinuria (CKD definition-1); (2) eGFR $<60 \mathrm{ml} \cdot \mathrm{min}^{-1} \cdot 1.73 \mathrm{~m}^{-2}$ (CKD definition-2).

\section{Measurements}

Blood samples were drawn from a peripheral vein while the subject was seated. When blood samples for routine blood testing were being taken, an additional $2 \mathrm{ml}$ was collected into a test tube containing EDTA-2Na for plasma BNP measurement. Tubes were stored immediately in an icebox after sampling and transported to the laboratory each afternoon where they were centrifuged at $1,500 \mathrm{~g}$ for $10 \mathrm{~min}$. After separation, plasma samples were stored frozen at $-20^{\circ} \mathrm{C}$ until transportation to the Shionogi central laboratory for assaying (Osaka, Japan). Plasma BNP levels were measured by direct radioimmunoassay using monoclonal antibodies specific for human BNP (Shiono RIA BNP kit, Shionogi). Cross-reac- tivity of the antibody was $100 \%$ for human BNP and $0.001 \%$ for human atrial natriuretic peptide. Intra- and interassay coefficients of variation were $5 \%$ and $6 \%$, respectively. Serum creatinine level was determined by an enzymatic method using an auto-analyzer (Hitachi 7700).

All subjects used a self-reported questionnaire to confirm their medical history, including status (yes/no) of prescribed drugs for hypertension, diabetes, hypercholesterolemia, stroke, angina, heart failure and myocardial infarction. Smoking status (current, past smoker or non-smoker) was also assessed by questionnaire.

\section{Risk Factor Definitions}

Systemic blood pressure was measured by experienced technicians. All subjects were seated for at least $5 \mathrm{~min}$ before measurement using an automatic device (BP-103i II, model 513000, Nippon Colin). Measurement was performed twice, with the mean value used for statistical analysis. Hypertension was defined as systolic blood pressure $\geq 140 \mathrm{mmHg}$ and/or diastolic blood pressure $\geq 90 \mathrm{mmHg}$ and/or the use of antihypertensive medication. Body mass index was calculated as weight $(\mathrm{kg})$ divided by the square of height $\left(\mathrm{m}^{2}\right)$. Diabetes was ascertained by non-fasting glucose concentration $\geq 200 \mathrm{mg} / \mathrm{dl}$ and/or hemoglobin A $1 \mathrm{c}$ value $\geq 6.5 \%$ and/or use of antidiabetic agents including insulin. Hypercholesterolemia was defined as a serum concentration $\geq 240 \mathrm{mg} / \mathrm{dl}$ and/or the use of antilipidemic medications.

\section{Outcome}

A follow-up survey assessing mortality, migration, and the incidence of heart failure, acute myocardial infarction and sudden death, and stroke was carried out after the baseline study. All deaths and migrations were confirmed by the official resident registration data issued by the local government offices.

Admission cases of heart failure in the cohort were checked by the regional registration survey data, which records primary hospital discharge diagnoses in the study area. The cases of heart failure were objectively defined by the Framingham criteria. ${ }^{16}$ Details of this register have been described previously. ${ }^{17}$ The event of non-sudden fatal myocardial infarction was also based on hospital registration survey data. The diagnosis of acute myocardial infarction was based on the Monica study criteria. ${ }^{18}$ Sudden cardiac death within $1 \mathrm{~h}$ of the onset of acute illness was examined using death records and then checked against medical records of the hospitals within the survey areas. Stroke registry was used for the outcome study. ${ }^{19}$ Stroke was defined as a sudden onset of focal neurological deficit $\geq 24 \mathrm{~h}$ duration and confirmed by brain computed tomography or magnetic resonance imaging.

\section{Statistical Analysis}

Continuous variables are shown as mean \pm SD. CKD subjects were divided into quartiles according to their baseline plasma BNP levels. To compare results among quartiles, ANOVA or chi-square test was used as appropriate. Survival from entry into the study was estimated using the KaplanMeier method, followed by a trend test (log rank). The association between baseline plasma BNP levels and endpoint $\mathrm{CV}$ diseases (new onset of heart failure, acute myocardial infarction/sudden cardiac death, and stroke) was evaluated. Using a Cox proportional hazards regression model, hazard ratios (HR) for plasma BNP with CV events were assessed. In this multivariable proportional-hazards regression model, 


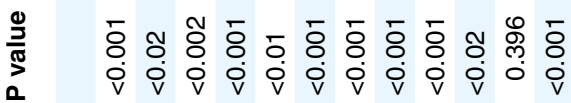

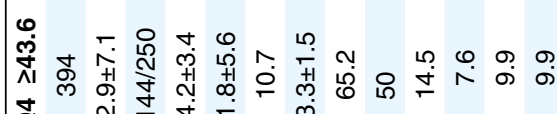

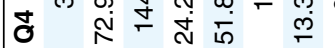

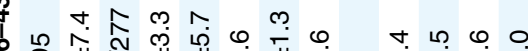
岗 $\stackrel{0}{0}$ ठㄷ लึ่ กิ

in

Z พิ่

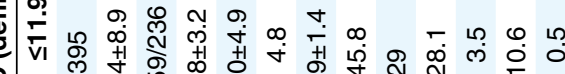
o

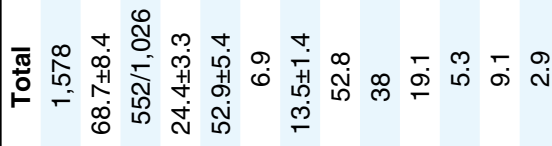

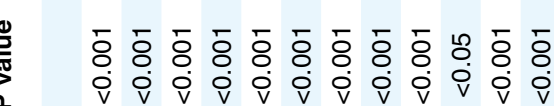

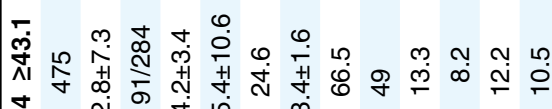

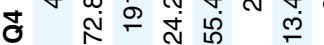

จัติ

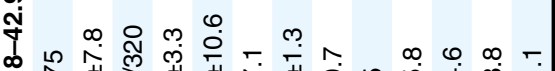
ฮ वृ

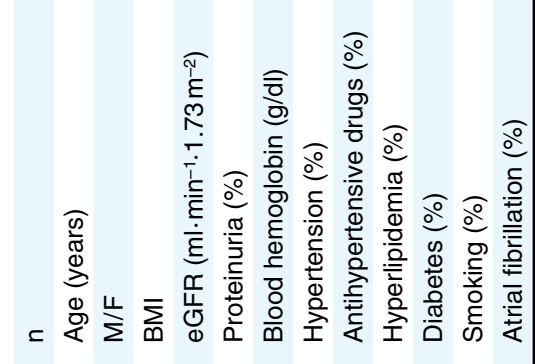

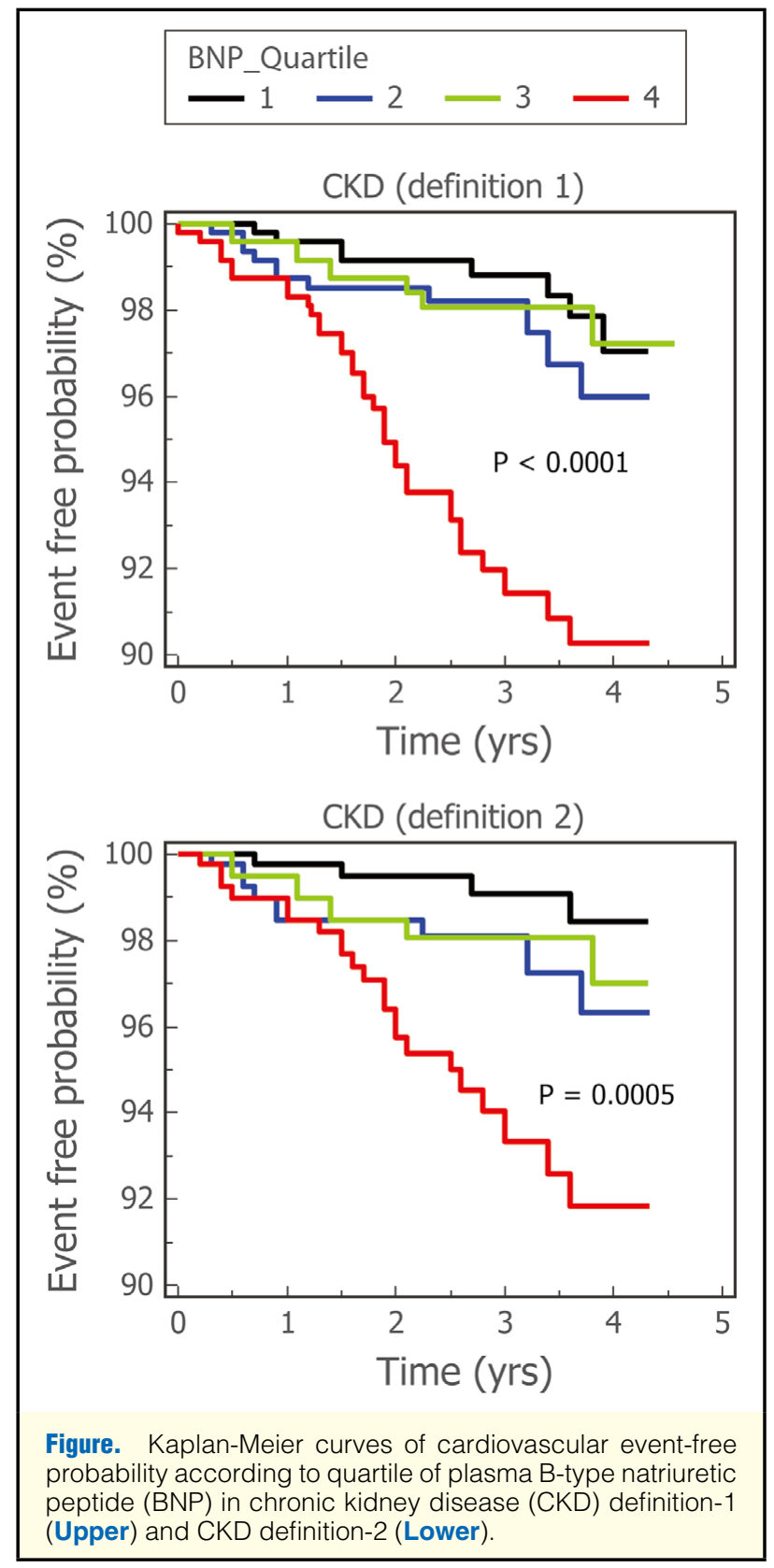

adjustments were made in the analyses for age, body mass index, and the presence or absence of hypertension, diabetes, hypercholesterolemia, current smoking, and atrial fibrillation. For analyses of CV incidence, person-years were censored at the date of $\mathrm{CV}$ events, the date of emigration from the study area, the date of death or the end of the follow-up period, whichever came first. All statistical analyses were performed using SPSS software (Chicago, IL, USA). A significant difference was defined as $\mathrm{P}<0.05$.

\section{Results}

As shown in Table 1, the number of cases of CKD definition-1 was 1,901 (727 in men, 1,174 in women). In this type of CKD, the prevalence within the community-based population was $14 \%$ ( $16 \%$ in men, $13 \%$ in women). The mean age was 67.9 years, and the mean eGFR was $57.4 \mathrm{ml} \cdot \mathrm{min}^{-1}$. 


\begin{tabular}{|c|c|c|c|c|c|}
\hline $\begin{array}{l}\text { BNP quartile } \\
\text { (pg/ml) }\end{array}$ & $\begin{array}{l}\text { All CVD events/ } \\
1,000 \text { person-years }\end{array}$ & $\begin{array}{c}\text { Age-sex adjusted } \\
\text { HR }(95 \% \mathrm{Cl})\end{array}$ & $P$ value & $\begin{array}{c}\text { Multivariate adjusted } \\
\mathrm{HR}^{\star}(95 \% \mathrm{Cl})\end{array}$ & $P$ value \\
\hline \multicolumn{6}{|l|}{ CKD (definition 1) } \\
\hline Q1 (s11.2) & 5.7 & 1.0 & & 1.0 & \\
\hline Q2 (11.3-22.7) & 8.6 & $1.77(0.70-4.49)$ & 0.226 & $1.83(0.72-4.66)$ & 0.203 \\
\hline Q3 (22.8-42.9) & 7.1 & $1.47(0.55-3.93)$ & 0.439 & $1.62(0.60-4.37)$ & 0.341 \\
\hline Q4 ( $\geq 43.1)$ & 25.9 & $4.71(2.04-10.90)$ & $<0.001$ & $4.59(1.97-10.73)$ & $<0.001$ \\
\hline \multicolumn{6}{|l|}{ CKD (definition 2) } \\
\hline Q1 (s11.9) & 3.5 & 1.0 & & 1.0 & \\
\hline Q2 (12.0-23.5) & 8.4 & $2.58(0.79-8.48)$ & 0.118 & $2.48(0.75-8.19)$ & 0.135 \\
\hline Q3 (23.6-43.4) & 7.7 & $2.39(0.70-8.12)$ & 0.164 & $2.56(0.75-8.73)$ & 0.134 \\
\hline Q4 ( $\geq 43.6)$ & 20.3 & $5.56(1.83-16.90)$ & $<0.003$ & $5.54(1.81-16.97)$ & $<0.003$ \\
\hline
\end{tabular}

*Adjusted for age, sex, BMI, current smoking, hypertension, diabetes, hypercholesterolemia, eGFR, and atrial fibrillation. $\mathrm{HR}$, hazard ratios; CVD, cardiovascular disease; $\mathrm{Cl}$, confidence interval. Other abbreviations see in Table 1.

$1.73 \mathrm{~m}^{-2}$. Proteinuria was found in $23 \%$ of the subjects. The percentages of cases of hypertension, diabetes, and atrial fibrillation were $54 \%, 7.5 \%$, and $3.1 \%$, respectively. The median plasma BNP level was $22.7 \mathrm{pg} / \mathrm{ml}$.

The number of cases of CKD definition-2 was 1,578 (552 in men, 1,026 in women), and the prevalence was $12 \%$ (12\% in men, $11 \%$ in women) within the community-based population. The percentages of hypertension, diabetes, and proteinuria were $53 \%, 5.3 \%$, and $6.9 \%$, respectively. The prevalence of atrial fibrillation was $2.9 \%$. The median plasma BNP level was $23.5 \mathrm{pg} / \mathrm{ml}$ (Table 1).

The cohorts were followed for 5,275 person-years in CKD definition-1, and for 4,350 person-years in CKD definition-2, respectively. Composite CV events (heart failure, acute myocardial infarction, sudden cardiac death, stroke) during the follow-up period (mean, 2.8 years) occurred in 62 cases in the CKD definition-1 group and in 43 cases in the CKD definition-2 group. The number of CV events/1,000 person-years was 11.7 and 9.9 in the CKD definition-1 and definition-2 groups, respectively.

Kaplan-Meier curves for the CV event-free rate according to the BNP quartiles in both CKD cohorts are shown in Figure. The CV event-free rate was significantly lower in the highest quartile of BNP $(>43 \mathrm{pg} / \mathrm{ml})$ in both CKD cohorts (CKD definition-1, $\mathrm{P}<0.0001$; CKD definition-2, $\mathrm{P}<0.0005$ by log-rank test).

As shown in Table 2, in the CKD definition-1 group, the number of CV events/1,000 person-years among BNP quartiles (Q) was 5.7 in Q1, 8.6 in Q2, 7.1 in Q3, and 25.9 in Q4. Similarly, in the CKD definition-2 group, the number was $3.5,8.4,7.7$, and 20.3, respectively. CV events occurred in the highest quartile group of each $\mathrm{CKD}$ cohort $(\mathrm{P}<0.001$ for both definitions).

After adjustment for age and sex, Cox regression analysis was performed to analyze the relationship between plasma BNP level and the risk of CV events (Table 2). The HR obtained from the Cox proportional model for the highest quartile of plasma BNP was significantly higher than that for the lowest quartile for CKD definition-1 (HR 4.71; 95\% confidence interval (CI) 2.04-10.9; $\mathrm{P}<0.001)$ and for $\mathrm{CKD}$ definition-2 (HR 5.56; 95\%CI 1.83-16.9; $\mathrm{P}<0.003$ ). In addition, after multivariate adjustment of the models (age, sex, BMI, smoking, hypertension, diabetes, hypercholesterolemia, atrial fibrillation, and eGFR), similar results were obtained (Table 2). The HR in the highest quartile was significantly higher compared with the lower quartile groups (HR 4.59 in
CKD definition-1 group, $\mathrm{P}<0.001$; HR 5.54 in CKD definition-2 group, $\mathrm{P}<0.003$ ).

\section{Discussion}

The present study demonstrates that for the first time in CKD cohorts defined by different criteria and selected from a community-based population, the subgroup with the highest plasma BNP quartile had a 4- to 5-fold higher CV risk, including heart failure, stroke, myocardial infarction, and sudden cardiac death compared with the subgroup with the lowest plasma BNP quartile. This relationship was robust even after adjustment for classical CV risk factors. Our observations suggest that the plasma BNP level is a useful tool for stratifying CV risk within a CKD cohort selected from a general population.

In cohort studies without renal dysfunction, Wang et al reported that the subgroup with plasma levels of BNP over the $80^{\text {th }}$ percentile had a 3-fold higher risk of new onset of heart failure and a 2-fold higher risk of brain transient ischemic attack than subjects showing plasma levels below the $80^{\text {th }}$ percentile. ${ }^{5}$ Similarly, in a general population without subjects with elevated serum creatinine levels, Kistorp et al demonstrated that subjects who had higher plasma NT-proBNP levels above the $80^{\text {th }}$ percentile had a 3 -fold higher risk of $\mathrm{CV}$ diseases than the subjects who had plasma NT-proBNP levels below the $80^{\text {th }}$ percentile. ${ }^{6}$ However, no studies have yet examined whether plasma levels of natriuretic peptides might be effective for stratifying the CV risk within a large number of CKD subjects selected from the general population. This may have been because of concerns that plasma natriuretic peptide levels might increase in the absence of organic cardiac disorders, and thus confound the relationship between the plasma level and CV events in this setting, as the important clearance site of the natriuretic peptide family protein is the kidney. ${ }^{20}$

There are several possible explanations for the fact that an elevated plasma BNP level was associated with a high risk for $\mathrm{CV}$ events, as demonstrated in the present study. First, the increased level of plasma BNP might be a marker for more advanced renal dysfunction, and deterioration of renal function is usually associated with an accumulation of traditional CV risk factors ${ }^{21}$ and there may be related increases in homocysteine, inflammation, oxidative stress, and thrombogenic factors. ${ }^{1,4}$ These factors may impair endothelial function, lead to progression of atherosclerosis, and thus increase 
the risk of CV events in CKD subjects. Second, the plasma BNP level has been reported as increased with progression of anemia, which is independent of the degree of cardiac dysfunction. ${ }^{22,23}$ In this regard, an elevated plasma BNP level may indicate advanced anemia, and thus be a marker at a high risk of $\mathrm{CV}$ events in $\mathrm{CKD}$ subjects. In fact, several reports have demonstrated that the prevalence of future onset of coronary artery disease and heart failure were significantly elevated in subjects with anemia. ${ }^{24-26}$ Third, elevated levels of plasma BNP denote impaired cardiac function, including latent structural heart diseases, cardiac volume overlord, and myocardial ischemia, and thus such patients are prone to $\mathrm{CV}$ disorders.

In the present study, although there were no significant differences in the levels of eGFR and blood hemoglobin between the $3^{\text {rd }}$ rd and the $4^{\text {th }}$ BNP quartiles, CV events were clearly prevalent in the highest quartile group. These findings indicate that the first and the second explanations are unlikely, and thus the third hypothesis may be the more possible. However, left ventricular function and morphological data were unavailable in the present cohort study, and it was unclear whether patients with structural heart disease or impaired cardiac function were more common in the $4^{\text {th }}$ quartile than in the lower quartiles. In previous studies using echocardiography, a plasma level of plasma BNP $>40-50 \mathrm{pg} / \mathrm{ml}$ was a useful marker with high sensitivity and specificity for identifying subjects with latent structural heart disease, including left ventricular dysfunction, valvular heart diseases, cardiomyopathy, and atrial fibrillation. ${ }^{27-29}$ In view of these findings, a CKD subgroup with elevated plasma BNP levels tends to show subclinical structural cardiac disorders and is associated with a high risk for heart failure, ischemic stroke, and coronary artery diseases. In accordance with this hypothesis, several reports have suggested that an increased plasma BNP level in patients with renal dysfunction is mainly caused by cardiac overload and intrinsic organic heart disease rather than renal dysfunction. ${ }^{30-32}$

Incidentally, the present study found that CKD definition1 using reduced GFR and/or proteinuria captured a greater number of subjects with CV events than CKD definition-2 using reduced GFR only (62 cases for definition- 1 vs 43 cases for definition-2). This observation suggests that definition-1 is more useful for the definition of CKD in terms of CV risk stratification. Measurement of 2 biomarkers (GFR and urinary protein) is therefore be recommended for the selection of CKD subjects within apparent healthy populations.

\section{Study Limitations}

Although the present study with its large sample size is a prospective community-based study that included routine biochemical data, several limitations must be considered when interpreting the results. More than $35 \%$ of the CKD subjects were receiving antihypertensive agents at baseline. Several types of antihypertensive drugs, such as angiotensin-converting enzyme-inhibitors and angiotensin II receptor blockers, reduce the onset of $\mathrm{CV}$ events. The present study did not evaluate the effects of these drugs on the incidence of $\mathrm{CV}$ events. However, the percentage of subjects receiving antihypertensive drugs increased with the quartiles of plasma BNP level (Table 1), which suggests that the CKD subjects with higher plasma BNP levels were likely to receive these medications. This limitation might have underestimated the association between plasma BNP level and CV events. The urine dipstick test used in the present CKD definition is usually regarded as not being accurate for the diagnosis of persistence proteinuria. However, in a previous populationbased study, trace proteinuria on dipstick test had good reproducibility and high sensitivity and specificity for detection of micro-albuminuria in an elderly population. ${ }^{33}$ In this regard, the inclusion criterion for $\mathrm{CKD}$ definition-1 in the present study was a trace result for dipstick test.

In conclusion, the plasma BNP level provides strong predictive information about the future onset of $\mathrm{CV}$ events in subjects with mildly reduced renal function. This result implies that plasma BNP measurement is a powerful tool for stratifying $\mathrm{CV}$ risk in CKD subjects selected from the general population.

\section{Acknowledgments}

This study was supported in part by the Japan Arteriosclerosis Prevention Fund, and grants-in-aid for the scientific research fund of the Ministry of Education, Science, and Culture of Japan (20590836). The authors thank the Northern Iwate Heart Disease Registry Consortium for permission to use regional registry data for heart failure.

\section{References}

1. Sarnak MJ, Levey AS, Schoolwerth AC, Coresh J, Culleton B, Hamm LL, et al. Kidney disease as a risk factor for development of cardiovascular disease: A statement from the American Heart Association Councils on Kidney in Cardiovascular Disease, High Blood Pressure Research, Clinical Cardiology, and Epidemiology and Prevention. Circulation 2003; 108: 2154-2169.

2. Zhang QL, Rothenbacher D. Prevalence of chronic kidney disease in population-based studies: Systematic review. BMC Public Health 2008; 8: 117.

3. Coresh J, Selvin E, Stevens LA, Manzi J, Kusek JW, Eggers P, et al. Prevalence of chronic kidney disease in the United States. JAMA 2007; 298: $2038-2047$.

4. Schiffrin EL, Lipman ML, Mann JF. Chronic kidney disease: Effects on the cardiovascular system. Circulation 2007; 116: 85-97.

5. Wang TJ, Larson MG, Levy D, Benjamin EJ, Leip EP, Omland T, et al. Plasma natriuretic peptide levels and the risk of cardiovascular events and death. $N$ Engl J Med 2004; 350: 655-663.

6. Kistorp C, Raymond I, Pedersen F, Gustafsson F, Faber J, Hildebrandt P. N-terminal pro-brain natriuretic peptide, C-reactive protein, and urinary albumin levels as predictors of mortality and cardiovascular events in older adults. JAMA 2005; 293: 16091616.

7. Tsutamoto T, Wada A, Sakai H, Ishikawa C, Tanaka T, Hayashi $\mathrm{M}$, et al. Relationship between renal function and plasma brain natriuretic peptide in patients with heart failure. J Am Coll Cardiol 2006; 47: 582-586.

8. Austin WJ, Bhalla V, Hernandez-Arce I, Isakson SR, Beede J, Clopton $\mathrm{P}$, et al. Correlation and prognostic utility of B-type natriuretic peptide and its amino-terminal fragment in patients with chronic kidney disease. Am J Clin Pathol 2006; 126: 506-512.

9. Ninomiya T, Kiyohara Y, Kubo M, Tanizaki Y, Doi Y, Okubo K, et al. Chronic kidney disease and cardiovascular disease in a general Japanese population: The Hisayama Study. Kidney Int 2005; 68: $228-236$.

10. Go AS, Chertow GM, Fan D, McCulloch CE, Hsu CY. Chronic kidney disease and the risks of death, cardiovascular events, and hospitalization. $N$ Engl J Med 2004; 351: 1296-1305.

11. Nakamura K, Okamura T, Hayakawa T, Kadowaki T, Kita Y, Ohnishi $\mathrm{H}$, et al. Chronic kidney disease is a risk factor for cardiovascular death in a community-based population in Japan: NIPPON DATA90. Circ J 2006; 70: 954-959.

12. Cirillo M, Lanti MP, Menotti A, Laurenzi M, Mancini M, Zanchetti A, et al. Definition of kidney dysfunction as a cardiovascular risk factor: Use of urinary albumin excretion and estimated glomerular filtration rate. Arch Intern Med 2008; 168: 617-624.

13. Ohsawa M, Itai K, Tanno K, Onoda T, Ogawa A, Nakamura M, et al. Cardiovascular risk factors in the Japanese northeastern rural population. Int J Cardiol 2009; 137: 226-235.

14. Makita S, Nakamura M, Satoh K, Tanaka F, Onoda T, Kawamura K, et al. Serum C-reactive protein levels can be used to predict future ischemic stroke and mortality in Japanese men from the general population. Atherosclerosis 2009; 204: 234-238.

15. Matsuo S, Imai E, Horio M, Yasuda Y, Tomita K, Nitta K, et al. Revised equations for estimated GFR from serum creatinine in 
Japan. Am J Kidney Dis 2009; 53: 982-992.

16. McKee PA, Castelli WP, McNamara PM, McKee PA, Feinleib M. The natural history of congestive heart failure: The Framingham study. N Engl J Med 1971; 285: 1441-1446.

17. Ogawa M, Tanaka F, Onoda T, Ohsawa M, Itai K, Sakai T, et al. A Community based epidemiological and clinical study of hospitalization of patients with congestive heart failure in northern Iwate, Japan. Circ J 2007; 71: 455-459.

18. Tunstall-Pedoe H, Kuulasmaa K, Amouyel P, Arveiler D, Rajakangas AM, Pajak A. Myocardial infarction and coronary deaths in the World Health Organization MONICA Project: Registration procedures, event rates, and case-fatality rates in 38 populations from 21 countries in four continents. Circulation 1994; 90: $583-612$.

19. Omama S, Yoshida Y, Ogawa A, Onoda T, Okayama A. Differences in circadian variation of cerebral infarction, intracerebral haemorrhage and subarachnoid haemorrhage by situation at onset. J Neurol Neurosurg Psychiatry 2006; 77: 1345-1349.

20. van Kimmenade RR, Januzzi JL Jr, Bakker JA, Houben AJ, Rennenberg R, Kroon AA, et al. Renal clearance of B-type natriuretic peptide and amino terminal pro-B-type natriuretic peptide a mechanistic study in hypertensive subjects. J Am Coll Cardiol 2009; 53: $884-890$.

21. Parikh NI, Hwang SJ, Larson MG, Meigs JB, Levy D, Fox CS. Cardiovascular disease risk factors in chronic kidney disease: Overall burden and rates of treatment and control. Arch Intern Med 2006; 166: $1884-1891$.

22. Fukuta H, Ohte N, Mukai S, Saeki T, Kobayashi K, Kimura G. Anemia is an independent predictor for elevated plasma levels of natriuretic peptides in patients undergoing cardiac catheterization for coronary artery disease. Circ J 2008; 72: 212-217.

23. Wold Knudsen C, Vik-Mo H, Omland T. Blood haemoglobin is an independent predictor of B-type natriuretic peptide (BNP). Clin Sci 2005; 109: 69-74.

24. Hamaguchi S, Tsuchihashi-Makaya M, Kinugawa S, Yokota T, Takeshita A, Yokoshiki H, et al; JCARE-CARD Investigators.
Anemia is an independent predictor of long-term adverse outcomes in patients hospitalized with heart failure in Japan: A Report From the Japanese Cardiac Registry of Heart Failure in Cardiology (JCARE-CARD). Circ J 2009; 73: 1901 - 1908.

25. Walker AM, Schneider G, Yeaw J, Nordstrom B, Robbins S, Pettitt D. Anemia as a predictor of cardiovascular events in patients with elevated serum creatinine. J Am Soc Nephrol 2006; 17: 2293-2298.

26. Sarnak MJ, Tighiouart H, Manjunath G, MacLeod B, Griffith J, Salem D, et al. Anemia as a risk factor for cardiovascular disease in The Atherosclerosis Risk in Communities (ARIC) study. $J$ Am Coll Cardiol 2002; 40: 27-33.

27. Nakamura M, Endo H, Nasu M, Arakawa N, Segawa T, Hiramori $\mathrm{K}$. Value of plasma B type natriuretic peptide measurement for heart disease screening in a Japanese population. Heart 2002; 87: $131-135$.

28. Niinuma H, Nakamura M, Hiramori K. Plasma B-type natriuretic peptide measurement in a multiphasic health screening program. Cardiology 1998; 90: 89-94.

29. Seki S, Tsurusaki T, Kasai T, Taniguchi I, Mochizuki S, Yoshimura M. Clinical significance of B-type natriuretic Peptide in the assessment of untreated hypertension. Circ J 2008; 72: 770-777.

30. Palmer SC, Richards AM. Does renal clearance differ between the B-type natriuretic peptides (BNP versus NT-proBNP)? J Am Coll Cardiol 2009; 53: 891-892.

31. Takami Y, Horio T, Iwashima Y, Takiuchi S, Kamide K, Yoshihara F, et al. Diagnostic and prognostic value of plasma brain natriuretic peptide in non-dialysis-dependent CRF. Am J Kidney Dis 2004; 44: $420-428$.

32. Sagnella GA. Measurement and significance of circulating natriuretic peptides in cardiovascular disease. Clin Sci 1998; 95: 519529.

33. Konta T, Hao Z, Takasaki S, Abiko H, Ishikawa M, Takahashi T, et al. Clinical utility of trace proteinuria for microalbuminuria screening in the general population. Clin Exp Nephrol 2007; 11: $51-55$. 